\title{
ON THE GROUPS OF ORDER $p^{m}$
}

\section{WHICH CONTAIN OPERATORS OF ORDER $p^{m-2 *}$}

BY

\section{G. A. MILLER}

Burnside has considered the groups of order $p^{m}$ ( $p$ being any prime) which contain an invariant cyclic subgroup of order $p^{m-2} \cdot \dagger$ Those in which a cyclic subgroup of order $p^{m-2}$ is transformed into itself by an abelian group of order $p^{m-1}$ and of type $(m-2,1)$ have also been studied. $\neq$ The main object of the present paper is to determine the remaining groups of order $p^{m}(m>4$ when $p$ is odd, and $m>5$ when $p=2$ ) which contain a cyclic subgroup of order $p^{m-2}$. As such a subgroup must be transformed into itself by $p^{m-1}$ operators of the group of order $p^{m}, \S$ each of these groups which does not come under one of the cases already considered must include the non-abelian group $H$ of order $p^{m-1}$ which contains $p$ cyclic subgroups of order $p^{m-2}$. The group of isomorphisms $(I)$ of $H$ is of order $p^{m-1}(p-1)$ and contains invariant operators of order $p^{m-3}$ when $p$ is odd and of order $p^{m-4}$ when $p=2$. $\|$

Let $P_{1}$ and $P_{2}$ represent two independent operators of $H$ whose orders are $p^{m-2}$ and $p$ respectively and let $P_{1}^{p^{m-3}}=P_{3}$. Suppose also that $P_{2}$ has been so chosen that $P_{2}^{-1} P_{1} P_{2}=P_{3} P_{1}$. The group of cogredient isomorphisms $\left(I_{2}\right)$ of $H$ is of order $p^{2}$ and of type $(1,1)$. When $p$ is odd $I$ includes an operator $\left(t_{1}\right)$ of order $p$ such that

$$
t_{1}^{-1} P_{1} t_{1}=P_{2} P_{1}, \quad t_{1}^{-1} P_{2} t_{1}=P_{2} .
$$

Since $t_{1}$ permutes the $p$ cyclic subgroups of order $p^{m-2}$ in $H$ cyclically, while some of the operators of $I_{2}$ are commutative with each operator of only one of these subgroups, the group generated by $I_{2}$ and $t_{1}$ is the non-abelian group of

* Presented to the Society (Chicago) January 3, 1902. Received for publication December $2,1901$.

† BURNSIDE, Theory of groups of finite order, 1897, p. 75.

$\ddagger$ Transactions of the American Mathematical Society, vol. 2 (1901), p. 259.

$\S$ BURnside, Proceedings of the London Mathematical Society, vol. 26 (1895), p. 209. Also, Frobenius, Berliner Sitzungsberichte (1895), p. 173.

$\|$ With respect to the non-cyclic group of order $p^{2}$, when $p$ is odd, or $p^{3}$, when $p$ is even, all the operators of a division have the same $p$ th power or $p^{2}$ th power respectively. Cf. Bulletin of the American Mathematical Society, vol. 7 (1901), p. 350 ; J. W. Young, Transactions of the American Mathematical Society, vol. 3 (1902), p. 189. 
order $p^{3}$ which contains no operators of order $p^{2}$. As this group contains only $p$ of the $p^{m-3}$ invariant operators of $I$ it follows that $I$ contains the nonabelian subgroup of order $p^{m-1}$ which includes no operator of order $p^{m-2}$ but has an invariant operator of order $p^{m-3}$, whenever $p$ is odd. This subgroup of order $p^{m-1}$ is invariant under $I$ according to Sylow's theorem. It is not difficult to see that the same group is invariant under the group of isomorphisms of the abelian group of type $(m-2,1)$.

\section{\$1. Determination of the groups when $p$ is even.}

When $p=2, I$ is of order $2^{m-1}$ and its subgroup $\left(I_{2}\right)$ which is composed of the group of cogredient isomorphisms of $H$ is the four-group. It includes an operator $t_{2}$ of order 2 such that

$$
t_{2}^{-1} P_{1} t_{2}=P_{1}^{-1}, \quad t_{2}^{-1} P_{2} t_{2}=P_{3} P_{2} .
$$

This operator is commutative with each operator of $I_{2}$ since it is evidently commutative with the operator $\left(t_{2}^{\prime}\right)$ which transforms $P_{1}$ into itself and $P_{2}$ into $P_{3} P_{2}$. Hence $I$ contains the abelian group of type $(m-4,1,1)$ and all the operators of this subgroup transform $P_{1}$ into a power of itself. An additional generator of $I$ is $t_{1}$ as defined above. It should however be observed that $t_{1}$ is commutative with only $p^{m-3}$ operators of $H$ when $p=2$, while it is commutative with $p^{m-2}$ of these operators when $p$ is odd.

It was observed above that $I$ contains an invariant operator of order $p^{m-4}$ when $p=2$. Let $t_{3}$ represent the operator of order 2 which is a power of this invariant operator. From the properties mentioned above it follows that

$$
t_{1}^{-1} t_{2}^{\prime} t_{1}=t_{3} t_{2}^{\prime}, \quad t_{1}^{-1} t_{2} t_{1}=t_{2}{ }^{*}
$$

Hence, when $p=2, I$ contains a subgroup of type $(m-4,1)$ which is composed of its invariant operators. It is completely defined by the fact that it contains such a subgroup and two non-commutative operators $\left(t_{1}, t_{2}^{\prime}\right)$ of order 2 with properties noted above.

We proceed to determine all the groups of order $2^{m}$ which contain $H$ and permute its cyclic subgroups of order $2^{m-2}$. Such a group must transform $H$ according to a subgroup of order 8 in $I$, which includes the group of cogredient isomorphisms of $H$. As all the operators of orders two and four contained in $I$ are included in its subgroup of order 32 there are just four such subgroups of order 8 and each of them is simply isomorphic with the octic group. $\dagger$ They are generated by $I_{2}$ and the following four operators of order two respectively:

$$
t_{1}, \quad t_{1} t_{2}, t_{1} t_{2}^{\prime} t_{4}, \quad t_{1} t_{2} t_{2}^{\prime} t_{4},
$$

* These equations may be verified by observing that each member transforms $P_{1}$ and $P_{2}$ in the same way.

† Cf. Pierpont, Annals of Mathematics, ser. 2, vol. 1 (1900), p. 140. 
where $t_{4}$ is an operator of order 4 in the group generated by an operator of order 8 in $I$.

The group $\left(G_{1}\right)$ generated by $H$ and $t_{1}$ contains just $2^{m-4}$ invariant operators and is conformal with the abelian group of type $(m-2,1)$; i. e., it contains $2^{a+1}$ operators of order $2^{a}(1<a<m-1)$ and 7 of order 2 . Its four cyclic subgroups of order $2^{m-2}$ involve, in pairs, the two cyclic subgroups of order $2^{m-3}$ contained in $H$. It follows directly from a known theorem that there is no other group which transforms $H$ in the way in which $G_{1}$ transforms it.*

The group $\left(G_{2}\right)$ generated by $H$ and $t_{1} t_{2}$ contains only 2 invariant operators. Its operators not contained in $H$ are composed of $2^{m-3}$ operators of order 2 and $3.2^{m-3}$ of order 4 . Since $P_{1}^{-2} t_{1} t_{2} P_{1}^{2}=P_{3} P_{1}^{-4} t_{1} t_{2}$ there can be no other group which transforms $H$ in the same manner as $t_{1} t_{2}$ does. Let $G_{2}^{\prime}$ represent the group generated by $H$ and $t_{1} t_{2}^{\prime} t_{4}$. Its $2^{m-3}$ invariant operators are generated by $P_{1}^{2}$ and it is conformal with $G_{1}$. As it contains an abelian subgroup of type $(m-2,1)$ it is not necessary to consider this group here. There is another group $\left(G_{3}\right)$ which transforms $H$ in the same way as $G_{2}^{\prime}$ does and contains four cyclic subgroups of order $2^{m-2}$. In $G_{3}$ all of these contain the same subgroup of order $2^{m-3}$ while this is not the case in $G_{2}^{\prime}$. Moreover, $G_{3}$ contains no operator of order 2 besides those in $H$ and it has no abelian subgroup of type $(m-2,1)$.

It remains to examine the case when $H$ is transformed in the same way as $t_{1} t_{2} t_{2}^{\prime} t_{4}$ transforms it. The group $\left(G_{4}\right)$ generated by $H$ and $t_{1} t_{2} t_{2}^{\prime} t_{4}$ contains only two invariant operators. Besides $H$ it contains $2^{m-2}$ operators of each of the orders 2 and 8 . In the other group $\left(G_{5}\right)$ which transforms $H$ in the same manner as $G_{4}$ does, there are $2^{m-2}$ operators of each of the orders 4 and 8 besides $H$. There cannot be more than two such groups, since $H$ has only two invariant operators under $G_{4}$. Hence there are just five groups of order $2^{m}$ which contain operators of order $2^{m-2}$ and in which no cyclic subgroup of this order is either invariant or transformed into itself by an abelian group of order $2^{m-1}$. It may be of interest to observe that the group of isomorphisms of $H$ when $p=2$ is identical with that of the abelian group of type $(m-2,1)$.

\section{§2. Determination of the groups when $p$ is odd.}

When $p>2$ the two sets of $p$ conjugate subgroups in $H$ are permuted by $I$ according to an intransitive substitution group of order $p^{2}(p-1)$, which is obtained by establishing a $(p, p)$ isomorphism between two metacyclic groups of degree $p$, just as in the case of the abelian group of type $(m-2,1) \cdot \dagger$ The

* Transactions of the American Mathematical Society, vol. 2 (1901), p. 265. The latter part of this theorem clearly assumes that $p$ is odd. It remains true, however, when $u_{1}$ is a power of $r_{1}$ and the order of $r_{1}$ is greater than 4. The general method explained in $\$ 2$ of the article cited is employed in the present article.

†l. c., p. 261. 
groups under consideration must transform the operators of $H$ according to a subgroup of $I$, which includes $I_{2}$, is of order $p^{3}$, and permutes the $p$ cyclic subgroups of highest order in $H$. It is evident that there are just $p$ such subgroups. They are non-abelian and $p-1$ of them include operators of order $p^{2}$.

To prove that these $p-1$ subgroups are conjugate under $I$ it seems desirable to employ some additional equations, which we proceed to develop. Let $t$ represent an invariant operator of order $p^{m-3}$ in $I$ and let $t^{m-5}=t_{4}$. It may be assumed without loss of generality that $t_{4}^{-1} P_{1} t_{4}=P_{1}^{1+p^{m-4}}$ and $t_{4}^{-1} P_{2} t_{4}=P_{2}$. There are $p(p-1)$ conjugates of $t_{1} t_{4}$ under $I$. They are

where

$$
t_{\alpha \beta}^{-1}\left(t_{1} t_{4}\right) t_{a \beta} \quad(a=1,2, \cdots, p-1 ; \beta=1,2, \cdots, p)
$$

$$
t_{\alpha \beta}^{-1} P_{1} t_{\alpha \beta}=P_{1}^{a}, \quad t_{\alpha \beta}^{-1} P_{2} t_{\alpha \beta}=P_{3}^{\beta} P_{2} \text {. }
$$

It follows that

$$
\left(t_{\alpha \beta}^{-1} t_{1} t_{4} t_{\alpha \beta}\right)^{-1} P_{1}^{a}\left(t_{\alpha \beta}^{-1} t_{1} t_{4} t_{\alpha \beta}\right)=P_{2} P_{1}^{a+a p^{m-4}+\beta p^{m-3}} .
$$

On the other hand

$$
\left(t_{1} t_{4}\right)^{-n} P_{1}^{a}\left(t_{1} t_{4}\right)^{n}=P_{2}^{a n} P_{1}^{a\left(1+p^{m-4}\right)^{n}+n p^{m-3} a(a-1) / 2}
$$

The right hand members of $(A)$ and $(B)$ are the same only if

$$
n=1+\beta p, \quad a=1 .
$$

Hence not more than $p$ of the $p(p-1)$ conjugates of $t_{1} t_{4}$ are powers of $t_{1} t_{4}$, i. e., the operators $t_{\alpha \beta}$ transform $\left\{t_{1} t_{4}\right\}$ into at least $p-1$ conjugate groups. It remains to observe that only one of these groups can be in any one $\left(I_{3}\right)$ of the $p-1$ subgroups of order $p^{3}$ under consideration.

The last fact follows readily from the isomorphism between $I$ and the given intransitive substitution group of order $p^{2}(p-1)$. In this isomorphism $I_{3}$ corresponds to the subgroup of order $p^{2}$ and $\left\{t_{1} t_{4}\right\}$ corresponds to an invariant subgroup of order $p$. The $I_{3}$ which includes $t_{1} t_{4}$ can therefore involve only $p$ of the conjugates of $t_{1} t_{4}$ under $I$. In other words, the conjugates of $t_{1} t_{4}$ are found in $p-1$ conjugates of $I_{3}$.

Since these $p-1$ subgroups of order $p^{3}$ are conjugate under $I$ it is necessary to consider only two cases, viz. : the one in which $H$ is transformed according to one of these $p-1$ subgroups and the other in which $H$ is transformed by the groups in question according to the subgroup of order $p^{3}$ in $I$, which includes no operator of order $p^{2}$. In the former case there are only $p^{m-4}$ invariant operators while each of the groups which belongs to the latter contains $p^{m-3}$ such operators. We proceed to prove that there is only one group $\left(G_{1}\right)$ which comes under the former case, while there are two $\left(G_{2}, G_{2}^{\prime}\right)$ which come under the 
latter. It is not difficult to see that the last one of these groups contains a subgroup of type $(m-2,1)$.

Let $t_{5}$ be an operator of order $p^{2}$ which transforms $H$ in the same way as $t_{1} t_{4}$ does and suppose that it has been so chosen that $t_{5}^{2}=P_{2}$. The group generated by $H$ and $t_{5}$ contains no operator of order $p$ besides those of $H$. That this is the only group in question which transforms $H$ in the same way as $G_{1}$ does may be proved in exactly the same manner as the theorem to which reference is made in the last footnote. It may be observed that $G_{1}$ is conformal with the abelian group of type $(m-2,2)$.

The group $\left(G_{2}\right)$ generated by $t_{1}$ and $H$ is conformal with the abelian group of type $(m-2,1,1)$. In fact, it includes the abelian group of type $(m-3,1,1)$ since $t_{1}$ is commutative both with $P_{1}^{2}$ and with $P_{2}$. The other group $G_{2}^{\prime}$, which transforms $H$ in the same manner as $G_{2}$ does, may be obtained by the method mentioned in the last footnote. Since it includes the abelian group of type $(m-2,1)$ it will not be considered here. Hence, there are two and only two groups of order $p^{m}(p>2$ and $m>5)$ which include operators of order $p^{m-2}$ without containing either an invariant cyclic subgroup of this order or an abelian subgroup of type $(m-2,1)$. These two groups are conformal respectively with the abelian groups of type $(m-2,2)$ and of type $(m-2,1,1)$. When $m=5$ the group $G_{1}$ evidently contains an invariant cyclic subgroup of order $p^{m-2}$; hence there is only one group of order $p^{5}(p>2)$ which contains operators of order $p^{3}$ without containing either an invariant cyclic subgroup of this order or the abelian group of type $(3,1)$. 